\title{
Nursing Analysis of Traumatic Spinal Injury in Emergency Nursing
}

\author{
Yanli Wu* \\ Qingzhen City Hospital of Traditional Chinese Medicine, Guiyang, Guizhou 551400, China
}

\begin{abstract}
Spinal injuries account for about $4.2 \%$ of the whole body fractures, usually occur in the young, middle-aged, the vast majority are caused by indirect forces, and direct violence is relatively rare. Spinal injury was common in T12 L1, followed by $\mathrm{C} 1-2$, C $5-7$, but there are about $20 \%$ spinal injury are multiple vertebral fractures. In addition to vertebral fractures, spinal injury is often associated with combined injury of accessory fracture, ligament rupture, etc. If associated with spinal cord injury, it may cause paraplegia. The purpose of this paper is to explore the nursing measures of traumatic spinal injury. In this paper, 134 cases of spinal injury patients adopted nursing measures were summarized. Results showed that among the 33 cases of type I patients, there was no case died in the emergency department or with increased neurological damage. There was also no case with aggravated neurological damage in 31 cases of type II patients, and finally smooth admission. In the 21 patients with type III, 7 patients had poor spine stability, and had a certain tendency of neurological damage, and then transferred to the department of orthopedics. 5 cases had delayed neurological symptoms and transferred to the department of orthopedics. The other 5 cases had no abnormal changes over 3 days observation and went home to conduct conservative treatment and got better results.
\end{abstract}

\author{
KEYWORDS \\ Spinal injury \\ Neurological symptoms \\ Emergency treatment \\ Complication
}

\section{Introduction}

Spinal injuries account for about $4.3 \%$ of the whole body fractures, usually occur in the young, middle-aged, the vast majority are caused by indirect forces, and direct violence is relatively rare. Spinal injury is common in T12 L1, followed by $\mathrm{C} 1 \sim 2, \mathrm{C} 5 \sim 7$, but there are about $20 \%$ spinal injury are multiple vertebral fractures. In addition to vertebral fractures, spinal injury is often associated

\section{Copyright ( $) 2016$ Yanli Wu}

doi: $10.18686 /$ aem.v5i3.9

Received: August 3, 2015; Accepted: September 5, 2015; Published online: September 12, 2015

This is an open-access article distributed under the terms of the Creative Commons Attribution Unported License (http://creativecommons.org/ licenses/by-nc/4.0/), which permits unrestricted use, distribution, and reproduction in any medium, provided the original work is properly cited.

${ }^{*}$ Corresponding author: Qingzhen City Hospital of Tranditional Chinese Medicine, Guiyang, Guizhou 551400, China. E-mail: wujiao009@sina.com with combined injury of accessory fracture, ligament rupture, etc. If associated with spinal cord injury, it may cause paraplegia. In addition to the correct treatment for traumatic spinal injury in clinic, the nursing work in the emergency department is also very important. The spine injury accounts for about $0.2 \% \sim 1.4 \%$, of which about $25 \%$ is spinal cord injury, whether the site treatment is effective will directly affect the development and treatment result of the disease. In the emergency nursing, effective protection of spine to prevent secondary injury is particularly important for preventing disability and improving rehabilitation rate. This article will discuss the emergency nursing of traumatic spinal injury.

\section{Clinical data}

In this group, there were 134 patients, 76 males and 58 females, aged from 80 to 19 years, with an average age of 38 years. There were 98 cases of single segment of vertebral injury, 36 cases of injury in two sections and above, there 
were 41 cases of combined spinal cord cauda equina injury, of which 21 cases were incomplete spinal injury, 7 cases were complete spinal injury, 12 cases were spinal cord concussion, 1 cases was cauda equina injury; there were 39 cases associated with injury of other parts of the body, of which 14 cases were brain injury, 10 cases were limb fractures, 5 cases of several united injury, 4 cases were abdominal solid organ injuries, 3 cases were blood gas and 3 cases were pelvic injury.

\section{Emergency nursing methods}

\subsection{Emergency treatment}

First, for spinal injury patients, the respiratory tract must keep unobstructed, especially facial first touchdown patients, oral cavity, nasal cavity and throat often have foreign matter, or blockage of these parts due to oral secretions, may cause difficulty in breathing, even suffocation and crisis life. Timely clean facial blood clots, dirt and secretion; open airway to prevent increased cervical vertebra injury. Therefore, the impurity or secreta of these parts should be cleaned in a timely manner to keep respiratory tract smooth and ensure patients to go hospital for treatment safely.

Second, careful, scientific carry should be made, especially in the case of suspected spinal injury, the correct scientific handling of the injury is crucial. Cardboard stretcher should be selected and 3 or more person should coordinate during carrying, up and down flatly, carefully bend, prohibit the patient's own activities, such as turning, standing, sitting up and so on, the moving personnel should stand on one side, one hold the injured area, flat on the hard stretcher, another gently pull head and stay in the middle position, place on a stretcher, both sides of the head are fixed with sandbags or other object, the raising head and activities of moving neck are prohibited. For patients with thoracic spine and lumbar injury, the proper handling is to send by cardboard stretche, absolutely prohibit carrying by back, dragging the shoulder, arm, picking up legs and other wrong practices, which will lead to spinal injury and increase disability of patients. If using canvas stretcher transmits the patient, must let patients take a prone position, to keep the spine straight, prohibit bending, to avoid aggravating the injury.

\subsection{Emergency nursing method}

\subsubsection{General emergency nursing}

(1) Must keep the ward clean and tidy, the beds should be smooth, dry and comfortable. Avoid dirty, chaotic, and poor; (2) Nurses should encourage patients to do active functional exercise, prevent muscle atrophy and joint stiffness, foot care bracket is used to prevent foot drop. (3) Pay attention to the patient's nutrition, adjust the diet, give high protein, high nutrition digestible food.

\subsubsection{Nursing of functional exercise}

Patients need to sleep on hard bed, a soft pillow cushion should be used at the part of fracture, so that the spine is straight, also enjoin patient to gradually conduct back muscle exercise after 3 days, according to patients condition, physical and mental state step by step. Specific method: (1) Support by the head, elbows and two legs, lift the waist, and repeat such practice; (2) One week after injury. On the basis of the above, use head and two legs to support and arms are kept in front of the chest, pick up the waist; (3) Prone position exercises: on the basis of above, stay in prone position and put the two hands behind the back, lift the head and neck, chest and leg at the same time by back muscles to leave the bed.

\subsubsection{Bedsore prevention and nursing}

In spinal injury patients, the occurrence rate of bedsore is $20 \% \sim 70 \%$. (1) Timing assisting patients to turn over, once every 2 hours, the prominent bone is massaged with alcohol 1 2 times a day; (2) Keep the bed flat, clean, dry, no wrinkles and make patients comfortable; (3) Pay attention to keeping the skin clean and dry, clean the skin with ethanol twice a day; and (4) For the long-term bedridden patients, air cushion ring, sponge pad and other should be placed at the compressed bone to mitigate local long-term compression.

\subsubsection{Patient's turn over nursing}

(1) When the patients are unable to turn over by themselves, the nurse should assist them. Method: one hand holds shoulder and another hand holds hip, both hands force upward and outward, patients change from supine position to lateral position, or from lateral position to supine position; (2) If the patients can turnover by themselves, they may do it, but the shoulders and pelvis must turn over together, not twist spine. (3) For the patients with skull traction, keep the head and trunk on the same plane, to prevent aggravation of damage or falling out of traction bow.

\subsubsection{Nursing of fecal incontinence and constipation}

Diet should be quantitative, cannot be more or less. Eat more crude fiber food, drink more water, can prevent dry stool. Reflex defecation training: wear gloves, expanding the anus of patients after breakfast every day can play a role to stimulate the anal sphincter, reflexly cause intestinal peristalsis, after the establishment of this reflex, pressing the anus by a finger can discharge feces. Along the colon direction, from up to down to massage the abdominal wall, it can stimulate the intestinal peristalsis and help defecation.

\subsubsection{Insist on carrying out the regular first aid knowledge training for the emergency medical personnel}

It can be led by the emergency center to organize relevant training courses, improve prehospital rescuers' awareness and level of emergency, in addition to strengthening 
schedule personal's systematic training of first aid skills, we should mainly train the medical staff on the emergency ambulance to master the fixed transportation of spinal injury patients and the other prehospital first aid skill, regularly conduct basic emergency knowledge and technical skills assessment and sudden walkthrough, in order to urge to learn and consolidate the knowledge, so as to ensure that the pre-hospital emergency rescue process smoothly.

\subsubsection{Strengthening psychological nursing}

Spinal injury patients are mostly young adults, sudden accident cause cruel blow to their families and themselves, and bring heavy burden to the family and society, they will have fear, anxiety, nervousness and other psychological reactions, even suicide, giving up treatment. We want to care for the patient and the family members, the introduction of successful medical records may obtain the cooperation of patients and their families, reduce their fears, enhance self-confidence, actively cooperate with the treatment, and encourage them to face life with a positive attitude.

\subsubsection{Social and public universal emergency training work}

As China's public emergency training is still in its infancy, some of the public lack basic medical knowledge, we need to popularize first aid knowledge and basic skills training [7]. In particular, strengthen special populations such as police, teachers, public transport division personnel, material management personnel's training. Guide family members or the first eyewitness to carry patients, reduce the rate of disability and mortality.

\section{Results}

Among the 36 cases of type I injury patients, there was no case died in the emergency department or with increased neurological damage. For 37 cases of type II patients, there was no case with increased neurological damage due to examination and carrying, all of them were successfully admitted to hospital. In 21 patients with type III injury, 6 cases had poor stability of spinal column and obvious neurological damage trend, treated by Department of orthopedics. 6 cases had delayed neurological symptoms and transferred to the department of orthopedics. Another 9 cases had no abnormal changes over 3 days observation. 40 cases of type IV injury patients are the same, went home for conservative treatment after nursing, regular outpatient review, and had a good prognosis.

\section{Discussion}

Trauma can lead to increased coma, and then increase spinal injury, it also brings difficulties to the early clinical rescue, diagnosis and treatment. In general, early emergency treatment need deal with the problem endangering life and safety of patients, may inadvertently ignore the spinal injury, thereby lead to increased illness or delay the timing of treatment of spinal nerve, therefore, we should strengthen the field emergency and professional delivery, require on-site treatment and delivery professionally and timely [4], and give early and professional medical treatment. In the course of transportation, strengthening the effective protection of injured site can also reduce the injury degree of spinal injury patients, although this study does not includes, the similar study shows that strengthening protection of the injured part during the movement process can reduce the spinal injury's degradation, we should strengthen the relevant personnel's transport knowledge and skills training, improve the protection awareness of injured part [5]. Although some studies showed that moving to the vehicle used by hospital has no significant effect on the degree of damage, however, the research results indicated that there were certain effects, the reasons may be that some relevant personnel of professional conveyance had unfamiliar spinal and spinal cord injury first-aid knowledge and skills, so the ambulances can transport in a timely manner, but failed to enhance the injury site protection accurately. As for nursing for patients with spinal surgery, master the prevention and treatment measures and nursing principles, take various effective against the various stages of the disease to reduce the patient's pain and discomfort, prevent and treat various abnormalities and complications. At the same time, the nursing staff should be earnest and responsible, mobilize the initiative of the patients and their families, recognize that from illness to rehabilitation is a long process. Therefore, in a sense, the nursing task of spinal surgery is more difficult.

\section{Reference}

1. Qiu HB, Chen Y. Primary study on emergency classification of spinal injury. Chinese Journal of Critical Care Medicine. 2001;21(12):725.

2. Li YC, Yu WB. Postoperative care of 1 patients with entire scalp avulsion, thoracic fractures and spinal cord injury. Chinese Journal of nursing. 2005;40(5):352-353.

3. Shi YQ. Nursing of the patients with spinal cord injury. Chinese Nursing Research. 2005;19(8):1549.

4. Chen GF. Emergency nursing of acute spinal injury. Fujian medical journal. 1999;21(2):111.

5. Li J. Early first aid and nursing of patients with spinal injury. Harbin Medical Journal. 2007;27(4).

6. Zhou XH. Emergency Nursing, version 1. Beijing: Science and Technology Press. 2003.

7. Yang LJ, Lu LL, Mi Q. Zhuhai prehospital emergency medical personnel service present situation and countermeasure. Chinese Medical Innovations. 2012;9(8):51-53. 